\title{
Morphological and Production Changes in Planktonic and Biofilm Cells Monitored Using SEM and Raman Spectroscopy
}

\author{
Kamila Hrubanova ${ }^{1}$, Vladislav Krzyzanek ${ }^{1}$, Ota Samek ${ }^{1}$, Radim Skoupy ${ }^{1}$, Martin Šiler ${ }^{1}$, Jan Ježek ${ }^{1}$, \\ Stanislav Obruča ${ }^{2}$ and Pavel Zemanek ${ }^{1}$ \\ ${ }^{1 .}$ Institute of Scientific instruments of the CAS, v.v.i., Brno, Czech Republic. \\ 2. Centre for Material Research, Brno University of Technology, Brno, Czech Republic.
}

Many microorganisms (e.g., bacteria, yeast, algae) are known to form a multilayered structure composed of cells and extracellular matrix. Such a formation is known as the biofilm. In this contribution we focus on the bacteria Burkholderia sacchari which accumulates biodegradable polyesters, namely the most common member of polyhydroxyalkanoates (PHAs), poly(3-hydroxybutyrate) (PHB). Therefore, we investigated planktonic cells and biofilm cells. Elucidation of the basic mechanisms of the biofilm formation and its influence on polyesters formation within the cells would contribute to deeper understanding of the basic biochemical mechanisms underlying this process and developing new and more efficient strategies for the utilization of bacteria for biotechnological applications.

In our investigations on planktonic and biofilm cells scanning electron microscopy (SEM) and Raman spectroscopy techniques were used. Here, SEM uses electron beam to gain information about morphology of cells which reflects cells response on the different cultivation conditions. Consequently, Raman spectroscopy [1] was used for the determination of polymer (PHB) present in the biomass. Thus, our study targets some factors which could lead to efficient industrial production of polymers in selected biotechnological production.

Bacterial culture of Burkholderia sacchari was cultivated in mineral media containing glucose (20 g/l) as the sole carbon source. The cultivation was performed in stirred $(80 \mathrm{rpm}) \mathrm{CDC}$ biofilm bioreactor, temperature was set at $30{ }^{\circ} \mathrm{C}$. After $72 \mathrm{~h}$ of cultivation, the biofilm cells were gently harvested from glass coupons and planktonic cells were collected by centrifugation ( $5000 \mathrm{rpm}, 5 \mathrm{~min}$.) and both cell types were subjected to analysis.

The samples of Burkholderia sacchari were cultivated as mentioned above and observed by cryo scanning electron microscopy (cryo-SEM). Both planktonic and biofilm cells were imaged using a cryoSEM. For freezing the propane-jet technique was used (JFD030; Bal-Tec, Balzers / Leica microsystems, Vienna). After freezing, the samples were mounted onto a double fracture cryo-stage under liquid nitrogen and transferred under inert gas in a cryo-high vacuum shuttle VCT100 (Leica microsystems, Vienna) to a pre-cooled freeze-fracture device ACE600 (Leica microsystems, Vienna) at $-140{ }^{\circ} \mathrm{C}$. The samples were then fractured and sublimated at $-95{ }^{\circ} \mathrm{C}$ for 5 minutes to remove deposited residual water condensation and ice crystals. Finally, they were transferred under high vacuum and low temperature using the shuttle VCT100 to a pre-cooled $\left(-120^{\circ} \mathrm{C}\right)$ cryo-SEM Magellan 400/L (FEI) for the imaging with $1 \mathrm{keV}$ electron beam and beam current $6.3 \mathrm{pA}$ without any metal coating. Our experimental results are presented in Figure 1 (SEM) and Figure 2 (Raman spectroscopy).

Based on our results presented here, further systematic studies are required in order to fully monitor mechanisms of PHB accumulation in planktonic and biofilm cultures. Such studies are currently under way in our laboratories, exploiting combination of SEM and Raman spectroscopy approaches. 
References:

[1] O. Samek et al, Sensors 10 (2010), p. 8635-8651.

[2] This work was supported by the Czech Science Foundation (GA15-20645S, GA16-12477S and GA17-15451S), the infrastructure by the Ministry of Education, Youth and Sports of the Czech Republic (LO1212), and the European Commission (ALISI No. CZ.1.05/2.1.00/01.0017).
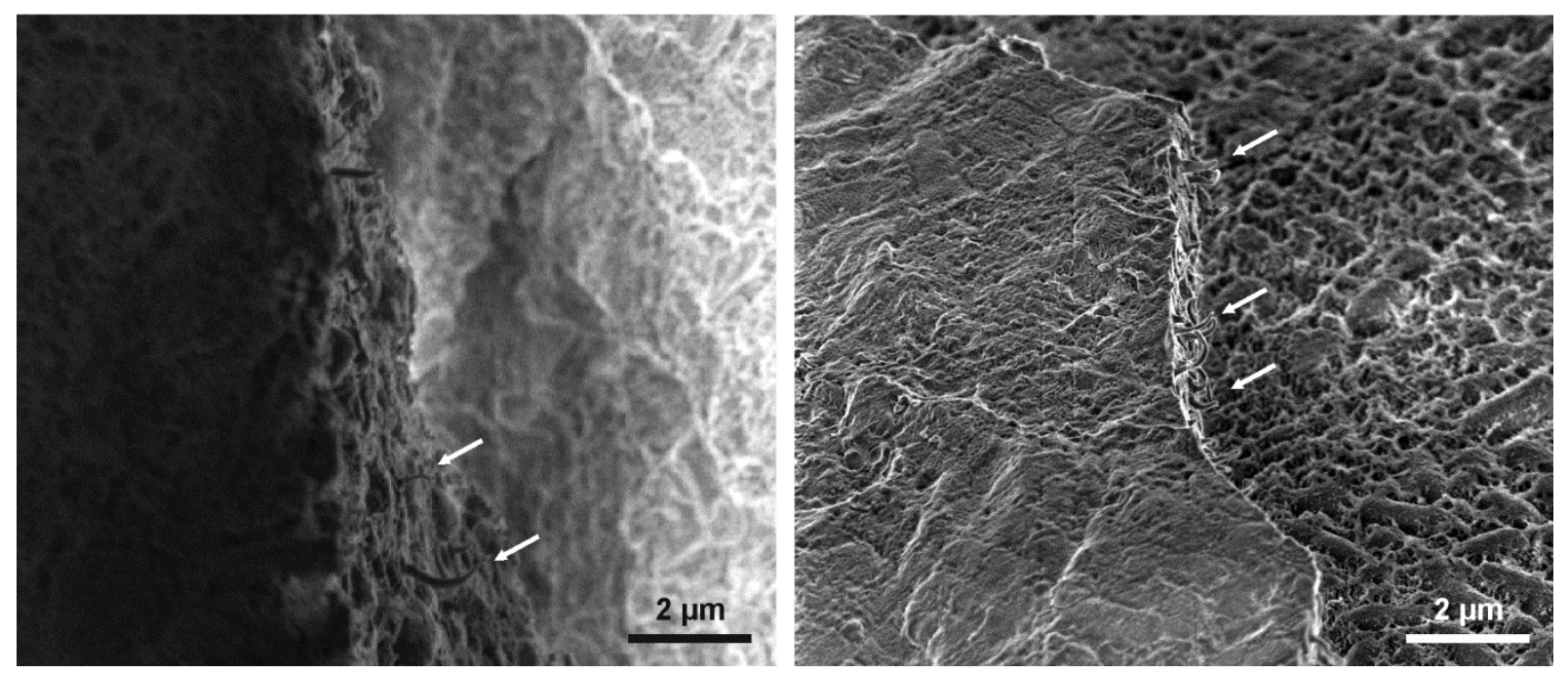

Figure 1. a) Cryo-SEM image of Burkholderia sacchari (biofilm cells); b) cryo-SEM image of Burkholderia sacchari (planktonic cells), here different surface morphology is clearly visible due to the higher content of cells with accumulated PHB (indicated by arrows).

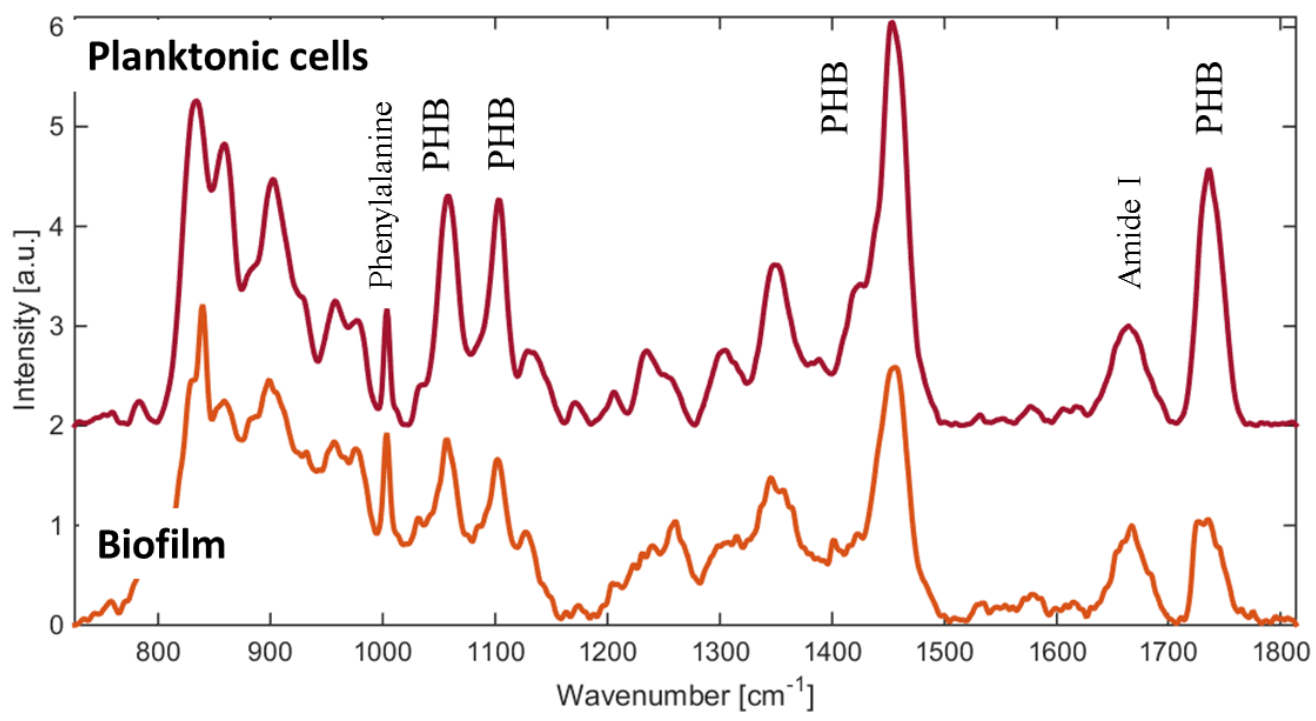

Figure 2. Typical Raman spectra of planktonic and biofilm cells (selected emission lines used in our study are highlighted) confirming findings of SEM - planktonic cells contain more PHB than biofilm cells. Cells were analyzed using a Renishaw Invia system (Renishaw inVia Raman Spectrometer, Renishaw plc., Wotton under Edge, UK), with a $785 \mathrm{~nm}$ single-mode diode laser as the excitation source. 